\title{
Research on Path Planning for Humanoid Robot based on Ant Colony Algorithm
}

\author{
ZHANG Xiaoliang ${ }^{1}$ \\ 1.School of Electronic and Information Engineering, \\ Ningbo University of Technology, Ningbo 315016 , \\ China; \\ zhangxiaoliang56@gmail.com
}

\begin{abstract}
In order to solve the complex problems in path planning of humanoid robot, an algorithm model based on ant colony algorithm is proposed. The robot simulation modeling environment is formed by the grid. The idea of the algorithm is simulating the process of ants foraging, and many ants complete optimal path search by collaborate with each other to pass the pheromone to find food. Through simulation experiments, we achieved better results. Satisfactory results are achieved by made experiments on the humanoid robot NAO, and the algorithm are verified finally.
\end{abstract}

Keywords: - humanoid robot; path planning; ant colony algorithm

\section{INTRODUCTION}

In recent years, robot develops quickly and is an integrated discipline which collection of computer, electron, machinery, sensor and other subjects, and humanoid robot gets more and more attention. Humanoid robot is a kind of robot which has humanoid form and humanoid behavior. Humanoid robot imitates humanoid inherent dynamic behavior from an engineering point of view, such as walking, function of study and so on [1]. With the development of humanoid robots research, path planning has been a very important part of humanoid robots research. The path planning is defined as: According to a certain evaluation criteria, look for a collision-free path which from initial state to target state in the environment with obstacles [2]. In path planning of humanoid robots, application of artificial intelligence algorithms is an important part of research. So far, many methods have been proposed for path planning of robot, such as free-space method, Petri algorithm, neural net algorithm [3], artificial potential field [4], fuzzy logic algorithm [5], genetic algorithms and $A *$ algorithm [6] and so on. These algorithms have their own advantages, but have some shortcomings either.

So the paper selects the ant colony algorithm which has strong robustness and ability of searching optimal path as algorithms for path planning. Most previous studies are only for mobile robot, in this paper, path planning based on ant colony algorithm be applied to humanoid robot, and do simulation experiment in a simulated environment, and do experiment using humanoid robot as its carrier for curve walking and avoiding obstacles in reality environment, and then complete the experiment for path planning of humanoid robot.

\author{
ZHONG Qiubo ${ }^{1,2}$ \\ 2. State Key Laboratory of Robotics and System, \\ Harbin Institute of Technology, Harbin 150001, China \\ zhongqiubo@gmail.com
}

\section{HUMANOID ROBOT PATH PLANNING BASED ON ANT COLONY ALGORITHM}

\section{A. The fundamental of ant colony algorithm}

Ant Colony Algorithm was proposed firstly by an Italy scholar M.Dorigo, etc [7]. It was a new algorithm proposed in 20th century to the 1990s. Ant Colony Algorithm was discovered by ants foraging. When people observe the process of ants foraging, it is easier for the colony of ants that cooperate with each other to find the shortest path to the source of food, but not for an ant [8]. Through further studied, we found that the ant could release a pheromone while walking. It is the pheromone that could be felt by ant. The concentration of that pheromone shows the distance of path. The higher concentration is, the shorter distance is. There is a good probability for ant to chose the path with higher concentration, and release some pheromones to increase the concentration of that pheromone at the same time, then the concentration of that pheromone will be higher. Meanwhile, the concentration will decline over time. Through this way, a favorable positive feedback formed.

The fundamental of Ant Colony Algorithm is showed such as Figure 1.It is supposed that Point A stands for nest, Point B stands for the souse of food and CF stands for the obstacle. The ant must through the BCF or EFD if it wants to walk from $\mathrm{A}$ to $\mathrm{B}$. To beginning, the probability is equal for the ant to chose. But the different distance of path leads to the different concentration of the pheromone. Finally, the colony of ants chose shorter path by the concentration.

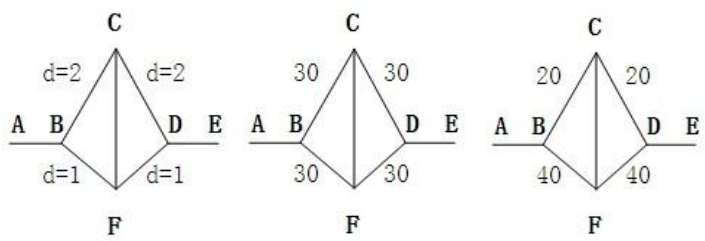

Figure 1. The basic principle of ant colony algorithm

\section{B. Ant colony algorithm}

Here we have an example about implementation process of ant colony algorithm in path planning. Suppose you want to use the ant algorithm to find a space in which an optimum path from the starting point to the target point.

The first step is using ant colony algorithm to find the path to the next node. In the literature [9], there are formulas have proposed node selection method and pheromone 
update, but it is still inadequate. For the treatment of pheromone is not ideal because pheromones will decline over time, and this formula can't be calculated Pheromone precisely. So in this paper, the formula has been added the time $t$ in order to deal with pheromone better.

When the time $\mathrm{t}$, the ant from the node $\mathrm{n}$ selects node probability of $\mathrm{m}$ :

$$
p_{n m}(t)=\frac{\tau_{n m}(t) * \eta_{n m}^{\beta}}{\sum_{x \in L} \tau_{n x}(t) * \eta_{n x}^{\beta}} \quad m \in L
$$

In the formula (1), $\tau_{n m}(t)$ represents pheromones from node $\mathrm{n}$ to node $\mathrm{m}, \eta_{n m}$ represents inspired information from node $\mathrm{n}$ to node $\mathrm{m}, \boldsymbol{\beta}$ represents the relative importance of inspired information, $L$ represent the optional nodes of next set.

Simultaneously, pheromone will be updated when an ant through the path. Ant passes will release the corresponding pheromone, but with the passage of time, the pheromone will gradually decline. For the initialization of pheromone in the literature [9] improves as:

$$
\tau_{n m}(t)=(1-\rho) \tau_{n m}(t)+\rho^{*} \tau_{0}
$$

In the formula (2), $\tau_{0}$ represents the initial value of the pheromone. $\rho$ represents the volatile factor of pheromone, what the range of $\rho$ is $[0,1]$. Then updating pheromone about the ants path. At time $\mathrm{t}+1$, we improve the pheromone formula in the literature [9] is:

$$
\tau_{n m}(t+1)=(1-\rho) \tau_{n m}(t)+\rho \Delta \tau_{n m}(t)
$$

In the formula (3), $\rho$ represents the volatile factor of pheromone, what the range of $\rho$ is $[0,1] \cdot \tau_{n m}(t)$ represents pheromones from node $\mathrm{n}$ to node $\mathrm{m}$. $\Delta \tau_{n m}(t)=1 / d_{n m}(t), d_{n m}(t)$ represents the shortest path between the node $\mathrm{n}$ and node $\mathrm{m}$ at time $\mathrm{t}$, the specific formula is:

$$
d_{n m}(t)=\sqrt{\left(x_{m}(t)-x_{n}(t)\right)^{2}+\left(y_{m}(t)-y_{n}(t)\right)^{2}}
$$

In the formula (4), $x_{n}$ represents the $\mathrm{x}$-axis coordinate values in node $\mathrm{n}$ at time $\mathrm{t}, x_{m}$ represents the $\mathrm{x}$-axis coordinate values in node $\mathrm{m}$ at time $\mathrm{t}, y_{n}$ represents the $\mathrm{y}$ axis coordinate values in node $\mathrm{n}$ at time $\mathrm{t}, y_{m}$ represents the $\mathrm{y}$-axis coordinate values in node $\mathrm{m}$ at time $\mathrm{t}$.

\section{The basic principle of humanoid robot path planning}

The path planning based on ant colony algorithm research object is humanoid robot. Thus we take NAO robot widely used at present as example. NAO robot is developed by the French Aldebaran Robotics, with higher intelligent.
In this article, we combine ultrasonic and visual to avoid obstacles to complete the path planning. The basic principle of path planning is as follows:

- First identify the size of pheromone concentration in the starting point, make the collected image gray processed, and extract the target to be recognized through the threshold processing, set the pixel value of output image that the pixel gray value of gray image below the threshold to black $(\mathrm{LOW}=0)$, then judge which side has higher pheromone, when there has no pheromones, randomly choose one side.

- And then choose walking pheromone is relatively high with larger probability, while ranging with ultrasonic, when $\mathrm{D}$ (distance between robot and obstacle) less than 0.3 , the output of instruction box is activated, NAO robot will not go forward.

- According to the data judge the position of the obstacles, if the obstacles right in front of the robot, it will call "Sonar Right Detected" event, let robot turn left to bypass the obstacles. At the same time, the path of the obstacles will increase corresponding pheromone.

Certainly, the noise is the biggest problem in the process of image processing in experiment. To solve this problem, we use Gaussian filter to smooth the image, Gaussian filter is essentially a kind of signal filter, which can take image data for energy conversion, and exclude the low energy that is the noise.

\section{The process of humanoid robot path planning}

Humanoid robot in the experiment of path planning, walking from the start point to the target, the specific path planning process is as follows:

a) Humanoid robot walk from the start point to the obstacles, while uninterruptedly judging the distance Dis1 with a certain range between humanoid robot and the obstacles.

b) When the distance Dis1 is detected to reach the minimum, NAO robot start obstacle avoidance.

c) When avoid the obstacles, pheromones will increase.

d) Continue to walk toward the target, and constantly detect the distance Dis 2 between humanoid and target, until the distance to the minimum.

e) After detect the target, NAO robot stop walking.

f) Repeat step (1), so as to simulate the effect of ant colony.

\section{EXPERIMENTS}

\section{A. Simulation}

We did simulation experiment in order to confirm the correctness and effectiveness of ant colony algorithm in path planning mentioned in this article. The parameters adopted are $\beta=2, \rho=0.3$. The robot simulated work environment is $15^{*} 15$ static grid environment, the black part that we select 
different grid randomly to generate on behalf of the obstacles, the white part on behalf of feasible region. Using the algorithm for path planning, the experiment results show that, if there is a feasible path, ant colony can find an optimal path after fewer search. The specific work environment is shown in figure 2:

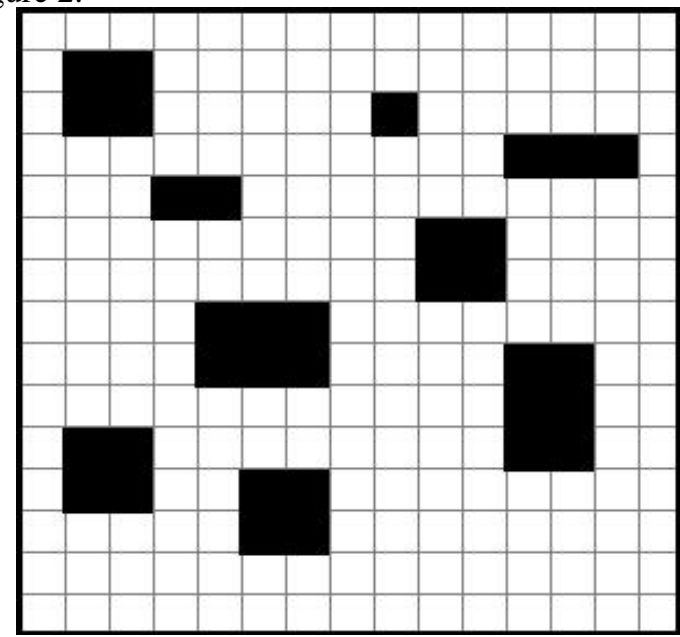

Figure 2. Work Environment

The robot needs to walk from the start point in the upper left corner to the target in the lower right corner. It adopt ant colony algorithm to find a collision less and optimal path from the start point to the target. The simulation results show the effectiveness and correctness of the algorithm. The simulation results as shown in figure 3 .

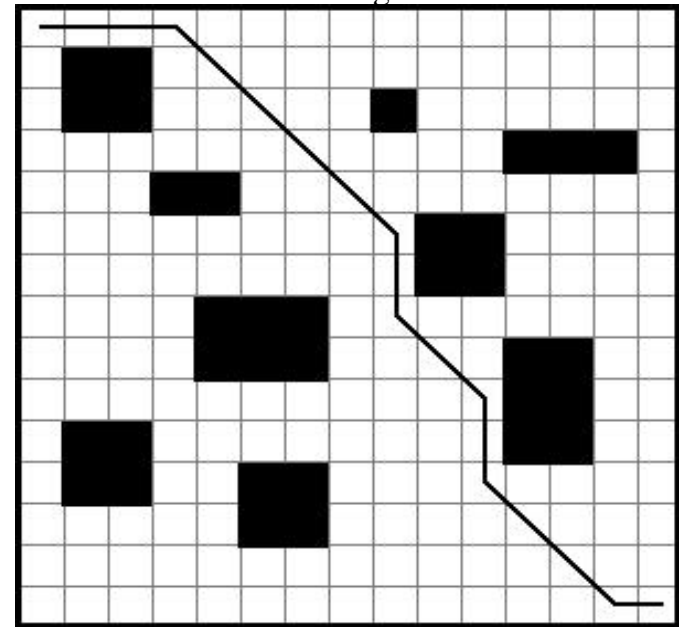

Figure 3. The simulation results

B. Curve walking and obstacle avoidance test of humanoid robot

This experiment used humanoid robot NAO. The robot has a dual channel ultrasonic sensor whose frequency is $40 \mathrm{KHz}$, sensitivity is $-86 \mathrm{~dB}$, resolution is $1 \mathrm{~cm}$ and effective cone is 60 degree. And NAO robot has two identical CMOS digital cameras, one of them located on NAO's forehead scans the horizon, another located at mouth level scans the immediate surroundings. The cameras' resolution is $640 * 480$, and it can capture up to 30 images per second, it can also upper and lower switch camera according to the need. Therefore it meets the requirement of this experiment.
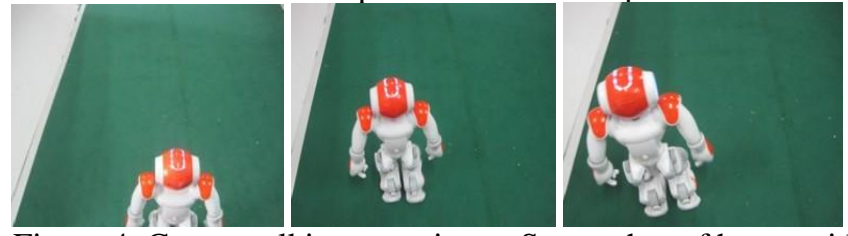

Figure 4. Curve walking experiment Screenshot of humanoid robot

The experimental results of curve walking show that NAO robot can successfully complete curve walking, as shown in figure 4 , then that experimental results can be applied to path planning. At the same time, the experimental results of obstacle avoidance show that NAO robot can successfully complete the process of obstacle avoidance, as shown in figure 5 , then can be applied to path planning with the curve walking experiment.
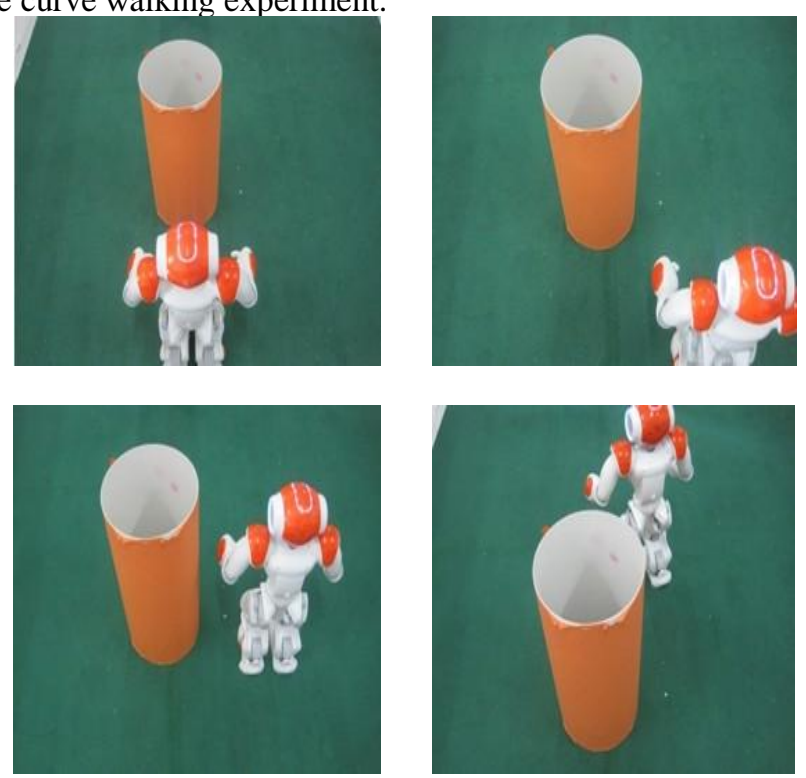

Figure 5. Obstacle avoidance test shots of Humanoid robot

\section{The experiment about path planning of humanoid robots}

The experiment about path planning was done in this paper. In this experiment, the experiment environment was idealized, there is no leaping obstacle, and the path is flat. It was be showed such as Graph 6.There are two path from the beginning to the end, sands for the left path and the right path respectively, and the left path is shorter than the right path. In this experiment, two NAO robots started from the same beginning, then they through the obstacles and go to the destination where there is a blue target. 

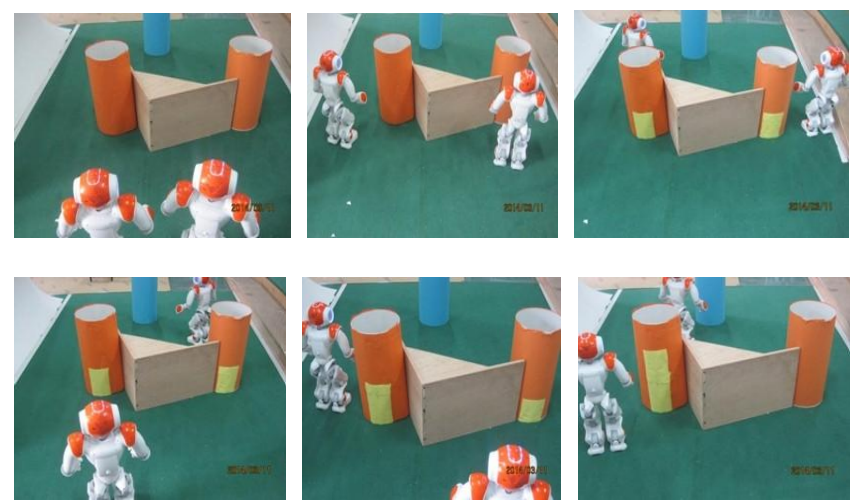

Figure 6.Path planning experiments Screenshot of Humanoid robot

When the NAO found the obstacle, it stopped walking and avoided the obstacles. When it avoided the obstacle, the concentration of the pheromone in the area where there is a obstacle would increase. The yellow stands for the pheromone in the picture. After avoiding the obstacles, NAO continue to walk until the distance from the destination get to a distance. The Graph 6 shows NAO which chose the right path stopped walking when arrived a distance which between NAO and the target. However, another NAO which chose the left path and arrived destination firstly had started from the beginning again.

After roundtrip many times, the pheromones of the left path is more than that of the right path, so the probability of NAO to chose the left path is more than to chose the right path. Keeping doing the experiment for a certain time, the pheromones of the right path became 0 , but the pheromones of the left path were much. Finally NAO chose the left path.

\section{CONCLUSION}

In this paper, ant colony algorithm is applied on a humanoid robot path planning, simulation and experiments of humanoid robot were done. The simulation results and experimental results of humanoid robot are verified the effectiveness and feasibility of the algorithm. But there are still many places to be studied and improved of ant colony algorithm.

\section{ACKNOWLEDGEMENT}

This material is based upon work funded by Natural Science Foundation of China under Grant No.61203360,
Zhejiang Provincial Natural Science Foundation of China under Grant No.LQ12F03001, LQ12D01001, LY12F01002, Ningbo City Natural Science Foundation of China under Grant No.2012A610009, 2012A610043, State Key Laboratory of Robotics and System (HIT)Foundation of China under Grant No.SKLRS-2012-MS-06, China Postdoctoral Science Foundation under Grant No.2013M531022

\section{REFERENCES}

[1] YU Xiuli,WEI Shimin, LIAO Qizheng. Explore the development and technology of Humanoid robot [J]. Journal of Mechanical Engineering, 2009, 45(3):71-75.(In Chinese)

[2] LI Qiang,CHEN Shanyong,YANG Shangang. Machine vision processing system based on TMS320VC5416 [J]. TV technology, 2004 , (7):79-82.(In Chinese)

[3] SONG Yong,LI Yibin,LI Chun,etc. Mobile robot path planning method based on neural network [J]. Systems Engineering and Electronics, 2008, 30(2):316-319.(In Chinese)

[4] SIllVA M O, SILVA W C, ROMERO R A F. Performance analysis of path planning techniques based on potential fields[C] Proceedings of the $7^{\text {th }}$ Latin American Robotics Symposium and Intelligent Robotics Meeting. Piscataway:IEEE Inc. Press,2010:115119.

[5] YANG Yipeng, LI Shaoyuan. Fuzzy optimization algorithm and its application in the planning path of robot [J].control and decision, 2007, $17:$ 727-726 (In Chinese)

[6] ZENG C,ZHANG Q,WEI X P. Robotic global path planning based modified genetic algorithm and $\mathrm{A}^{*}$ algorithm $[\mathrm{C}]$. Proceedings of the $3^{\text {rd }}$ International Conference on Measuring Technology and Mechatronics Automation. Piscataway:IEEE Inc,Press,2011:167-170.

[7] Marco Dorigo,Gianni Di Caro,Luca M Gambardella. Ant algorithms for Discrete Optimization. Massachusetts Institute of Technology,Artificial Life, 1999:137-172.

[8] GAO Wei,YIN Zhixi. Modern intelligent bionic algorithm and its application[M]. BEIJING: Science Press,2011,118-120.(In Chinese)

[9] SHI Feng,WANG Hui,Yu Lei,Hu Fei. MATLAB intelligent algorithm 30 case studies [M]. BEIJING: Beijing University of Aeronautics and Astronautics Press, 2011, 220-222.(In Chinese) 